\title{
GENERATION OF STATIC AND DYNAMIC SMALL CALIBRATION FORCES AND THEIR MEASUREMENTS BY ELECTROMAGNETIC FORCE COMPENSATION BALANCE
}

\author{
S. Vasilyan ${ }^{1}$, N. Rogge ${ }^{1}$, E. Manske ${ }^{1}$, T. Fröhlich ${ }^{1}$ \\ ${ }^{1}$ Institute of Process Measurement and Sensor Technology, Ilmenau, Germany, suren.vasilyan@tu-ilmenau.de
}

\begin{abstract}
:
The paper presents some of the results of the static and dynamic force measurements at $100 \mathrm{nN}$ to sub- $10 \mu \mathrm{N}$ ranges which are generated due the photon-momentum. The force sensor with resolution about $20 \mathrm{nN}$ and operating in differential measurement mode is developed by two electromagnetic force compensation balances. In order to generate these calibration forces, $\mathrm{CW}$ lasers with different operational modes, power levels, and wavelengths are used. Multi-reflection configuration of the laser beam inside the macroscopic cavity with highly reflective mirrors are used to test and variate the total amount of the forces.
\end{abstract}

Keywords: photon momentum, static and dynamic small calibration forces, high power laser, highly reflective mirrors

\section{INTRODUCTION}

In recent years, the use of photon momentum to determine the optical power of lasers or to generate precision/calibration small forces $[1,2,3]$ has made important progress, especially for the measurements of optical laser power at kilowatt levels [4]. The measuring principle is based on the measurement of the force which is exerted due to the transfer of the photon momentum upon reflection of the radiant power from a highly reflective mirror. A measurement device developed by Williams et al. [4] uses this measurement technique and achieves a relative expanded measurement uncertainty of $1.6 \%$ for optical power levels between $1 \mathrm{~kW}$ and $50 \mathrm{~kW}$. In the core of the device is a force sensor, consisting of a commercial off-theshelf electromagnetic force compensation (EMFC) balance and a mirror with high reflectivity $(R=0.9998 \%)$ attached to it. Vasilyan et al. $[1,2]$ carried out measurements with a device having similar components, however, here two force sensors adapted for differential measurements were used, by which the noise level has been reduced. Here, for the stability considerations a low power laser system (around $1 \mathrm{~W}$ ) was used in multireflection configuration to generate calibration forces at the currently existing lowest end of the small force standard typically referred to be from $10 \mathrm{nN}$ up to $10 \mu \mathrm{N}$. Despite the statistical error observed as an oversight between the measurements and simplified theoretical calculations, under the multi-reflection configuration the total measured force was amplified by at least an order of magnitude in comparison to the single reflection configuration. Furthermore, with the usage of single- and multi-reflection configurations, a possible standard for the force calibration routine, or reversed, standard for the optical (laser) power calibration routine with direct and more simplified traceable chain to the recently renewed SI base units $[2,5,6]$ was already established.

\section{DESCRIPTION OF THE WORK}

In order to develop a proper methodology in using this effect of the photon momentum-generated small calibration forces, initially several test measurements have been carried out. The setup consists of two EMFC balances operating in synchronous measurement modes such that the difference signal can be obtained with substantially reduced noise level.

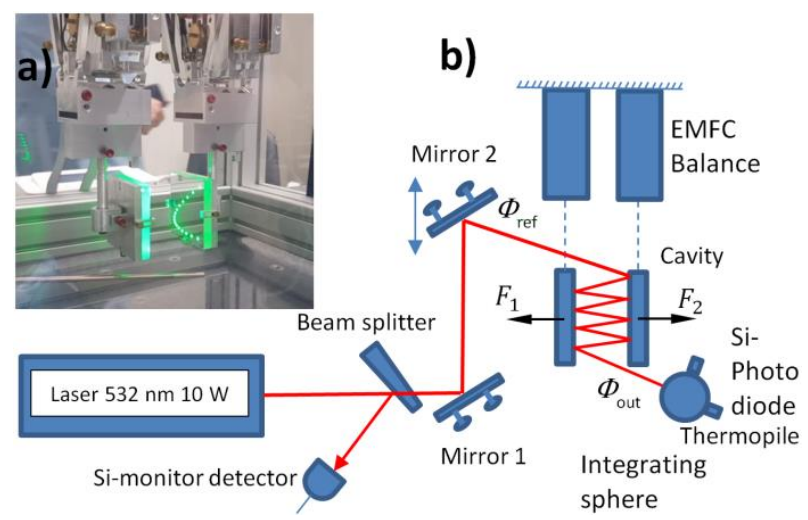

Figure 1: Set-up for measuring the generated small calibration forces using EMFC balance, the supporting optical components and the reference optical power sensors. Inset the photograph showing the lower parts of the EMFC balances, mirrors and the reflection spots 
The EMFC balances are mounted independently from the common metrology frame and on each of them a highly reflective mirror is attached with total weight of about $70 \mathrm{~g}$ together with the adjustment mechanism (see scheme in Figure 1). A conventional off-the-shelf highly reflective mirror with reflectivity $R$ above 0.995 , universally used for lasers with different wavelengths (see Table 1), has been used to create the macroscopic optical cavity where the multi-reflections of the laser beam are obtained.

Table 1: Summary of the parameters of the laser (power $P$, power uncertainty $U$, wavelength $\lambda$ ) and the mirror reflectivity coefficient $R$ used during different measurements

\begin{tabular}{|c|c|c|c|l|}
\hline & \multicolumn{3}{|c|}{ Laser } & \multicolumn{1}{c|}{} \\
\cline { 2 - 5 } & $\boldsymbol{P} / \mathbf{W}$ & $\boldsymbol{U} / \boldsymbol{\%}$ & $\boldsymbol{\lambda} / \mathbf{n m}$ & \\
\hline 1 & 0.98 & 1 & $420 \pm 10$ & 0.999 \\
\hline 2 & $0.05-2$ & 1 & $450 \pm 7$ & 0.999 \\
\hline 3 & $0.1-0.9$ & 1 & $520 \pm 2$ & 0.995 \\
\hline 4 & $2-11$ & $<1$ & $532 \pm 5$ & 0.995 \\
\hline 5 & $2.5-8.8$ & $<1$ & $532 \pm 5$ & 0.99997 \\
\hline
\end{tabular}

Theoretically, at the single reflection of the laser beam with $1 \mathrm{~W}$ optical power from a perfectly reflecting mirror, a force of about $6.7 \mathrm{nN}$ is generated. In the case of the multiple reflecting specular type of reflections, a force of about $220 \mathrm{nN}$ can be created, see equations (1) and (2).

$$
\begin{gathered}
F=\frac{P}{c}(1+R) \\
\sum_{j=1}^{n} F_{j}=\frac{(1+R)}{c} \sum_{j=1}^{n} P_{j}
\end{gathered}
$$

where $F$ is the force in $\mathrm{N}, P$ is the average laser power in $\mathrm{W}, c$ is the speed of light in $\mathrm{m} / \mathrm{s}, R$ is the reflectivity coefficient of the mirror, and $j$ is an integer assigned to each of the $n$ reflections.

During the measurements the photon momentum-generated forces due to specular type of multi- reflection are described in equation (3).

$$
\begin{aligned}
F_{\text {total }} & =F_{1}-F_{2}= \\
& =\left[F_{\mathrm{P} 1}+F_{\mathrm{Err} 1}\right]-\left[-F_{\mathrm{P} 2}+F_{\mathrm{Err} 2}\right]
\end{aligned}
$$

where $F_{\mathrm{P} 1}$ and $F_{\mathrm{P} 2}$ are the photon momentumgenerated forces acting on the EMFC balances attached left and right mirrors from 2nd, 4th, 6th... and 1 st, 3 rd, 5 th $\ldots$ reflections respectively, $F_{\text {Err1 }}$ and $F_{\text {Err2 }}$ are the noise measured by each EMFC balance which are assumed to be the same (see Figure 1).

The parameters listed in Table 1 were used during different measurement campaigns. The averaged laser power has been pre-set using varying methods. We have separated dynamically changing and statically applied laser powers in order to characterise the force measurement signals in relation to them, more specifically in the time or frequency domains. In the case of the static signals have been used either a shutter system or completely turning the lasers off. In the case of the dynamic signals the power of the laser has been varied by varying the gain current of the laser's control unit using equation (4).

$$
\begin{aligned}
& P \sim I_{i}\left(f_{i}\right)=\Delta I \sin \left(2 \pi f_{i} \Delta t_{i}\right)= \\
& =\Delta I \sin \left(2 \pi\left[f_{0}+i \cdot \Delta f\right] \Delta t_{i}\right)
\end{aligned}
$$

where $\Delta I$ is the peak-to-peak amplitude of the applied gain current, within the frequency range $f_{0}$ to $f_{\text {end }}$, with $\Delta f$ separation between the grid points, and $\Delta t_{i}$ is the signal length. Any other function type could be used, e.g. a square wave (see Figure 2).

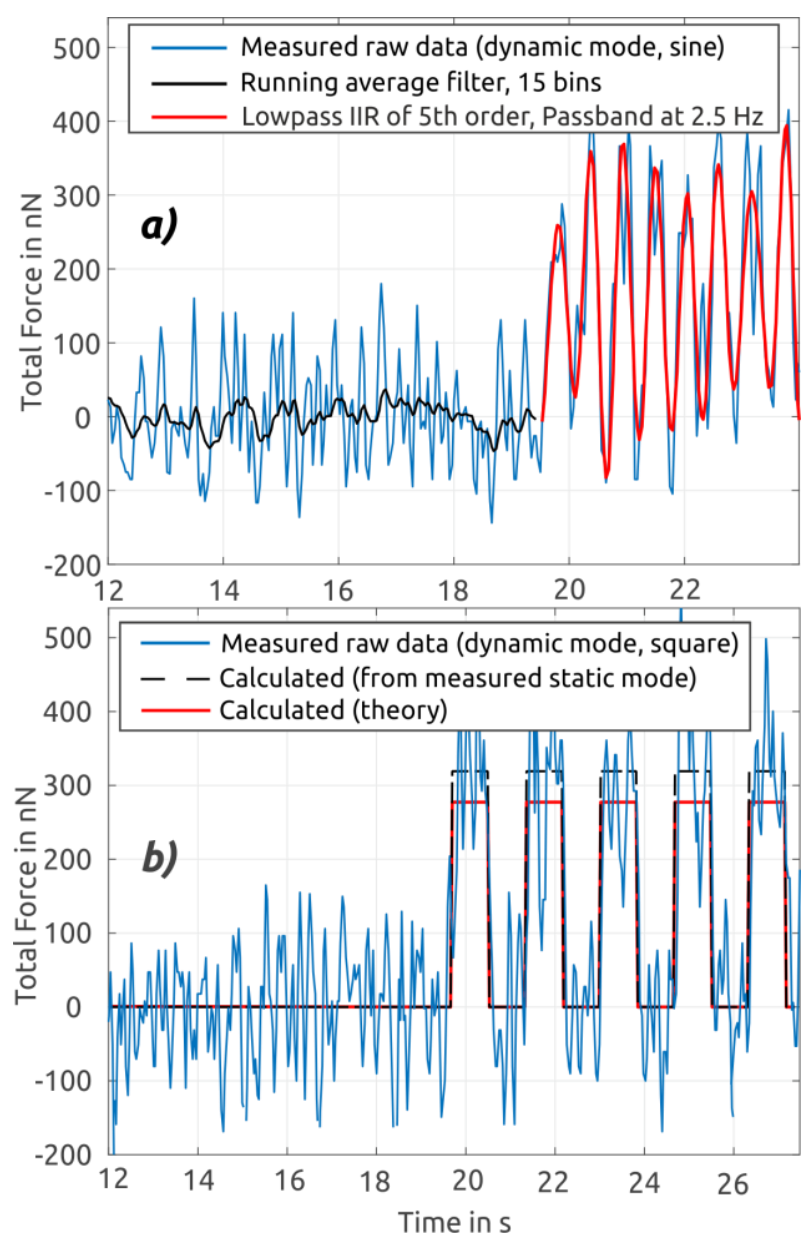

Figure 2: Example of the measured forces as a) sine and b) square wave functions [2]

\section{UNCERTAINTY ASSESSMENT}

The photon momentum-based method, as mentioned, may directly relate the measure of the force, which is produced by the momentum transfer of the absorbed and re-emitted photons from a highly reflective mirror, with the measure of the optical power of a laser beam. The following simplified numerical example, based on equating the photon momentum-generated forces with the 
gravitational force acting on a standard mass piece, gives an understanding of the advantages underlying this method. We should note here beforehand that as it is known from recent developments the mass pieces mainly at $1 \mathrm{~kg}$ and $500 \mathrm{~g}$ are already calibrated directly traceable to the quantum-based natural constants, more specifically it is traceable to the numerical value of the Planck constant, however there are still ongoing developments to extend that calibration method of mass pieces for the range of smaller mass pieces (down to $1 \mathrm{mg}$ ) [6]. Using the Kibble balance apparatus, one may even provide calibration of the arbitrary values of mass pieces, therefore on the account of measuring the gravitational force acting to this standard mass pieces one may also provide static force calibration values with similar accuracy and precision. Here we will rather provide our analysis on the basis of existing basic specifications of the measurement components that are well known/established and at the same time assumes the future speculations related to possible approaches of calibrating forces at smaller force levels.

It is known that the optical power measurements at $1 \mathrm{~kW}$ level are typically made using reference standard thermal detectors with several percent of uncertainty, at the same time becoming a non-trivial technological task to implement since their measurement capability and accuracy strongly depend on the absorbance and heat capacity of the cavity used as sensor. In comparison to this, the $1 \mathrm{~kW}$ laser power after first reflection from the ultra-high reflective mirror with 0.99995 reflectivity exerts a force of about $6.673116 \mu \mathrm{N}$, whereas after 33 reflections it is $220.03676 \mu \mathrm{N}$. One may use standard practices known from mass/force metrology to perform the force measurements at these levels. In the case that one uses a commercially available precision balance with $3 \mu \mathrm{g}$ reproducibility (standard deviation) in measurements of the weight pieces, the equivalent force calculated with equation (2) is to be $29.4 \mathrm{nN}$ assuming the value for the local gravitational acceleration of $9.812503 \mathrm{~m} \cdot \mathrm{s}^{-2}$ at the site of the measurements. Thus, in this case we can measure the optical power generated forces after first reflection with $0.440 \%$ relative standard deviation and with $0.013 \%$ after 33 reflections. However, since force standards at the nanonewton level have not yet been established, the implementation of a traceable optical power measurement using the photon momentum approach can only be verified in connection to the measurement capabilities of a certain class of apparatuses, e.g. with an electromagnetic force compensation - EMFC weighing balances down to $10 \mu \mathrm{N}$ (with about $0.3 \%$ relative measurement uncertainty) traceable from there down to about $10 \mathrm{nN}$ only accounting for the reproducibility or in general the Type $B$ uncertainty evaluation (an alternative to be the development of an uniquely-designed custom-made instrument with well characterised and SI-traceable calibration certificates whose performance could be verified by an independent party).

Generally, the balances are calibrated by employing the deadweight effect exerted on standard weight pieces in accordance with the equation $F=m g$. On the other hand, the optical power can be determined by combining this with equations (1) and (2) for single or multiple reflection cases yielding the simplified form given in equation (5).

$$
P=c \frac{m g}{1+R_{L}}
$$

from which, by using the standard uncertainty propagation, we obtain equation (6).

$$
\frac{u(P)}{P}=\sqrt{\left(\frac{u(m)}{m}\right)^{2}+\left(\frac{u(g)}{g}\right)^{2}+\left(\frac{u\left(R_{L}\right)}{R_{L}}\right)^{2}}
$$

The value of the gravitational acceleration can typically be obtained to better than $0.2 \mathrm{ppm}$. The values of the reflectivity for the ultra-high reflective mirrors to our best knowledge can be estimated only indirectly by the measurement of the losses typically given as the optical transmission curve. In accordance to most of the datasheets provided by manufacturers it can be determined with $70 \mathrm{ppm}$ uncertainty [9]. The uncertainty values of the mass vary dependent from the nominally used discrete set of standard mass piece (ref. Figure 3) against which the nominally applied magnitude of the optical power should be compared. For this, one should follow at least the recommendations given in [8].

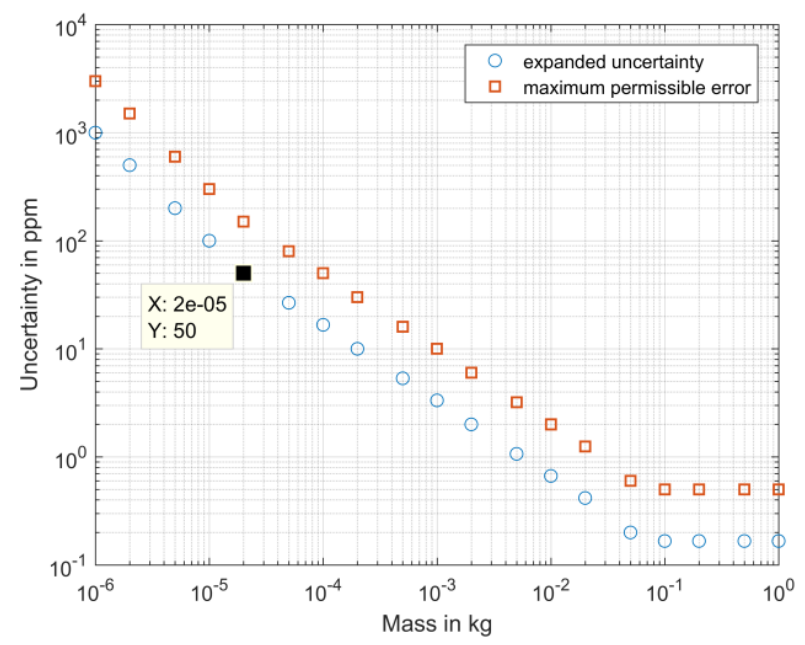

Figure 3: Expanded relative uncertainties and maximum permissible errors associated with weights of OIML R 111-1 class $\mathrm{E}_{1}$ [8]

Thus, if the measurements would be conducted at the $1 \mathrm{~kW}$ optical power level for the single reflection case the combination of uncertainty 
contributions using equation(6) leads to an estimated standard uncertainty of the optical power measurements of about $u(P) / P=1002 \mathrm{ppm}$; for the multi-reflection configuration with 33 reflections the value is $86 \mathrm{ppm}$. In Figure 4 the full estimation curves of relative measurement uncertainties in the optical power obtained by equation (6) are presented. Here the relative standard uncertainty is presented as a function of the nominal magnitude of the applied optical power in four different $(1,3,21,33)$ reflection configuration cases with interpolated data as an extended estimate for the power levels below $1 \mathrm{~kW}$. The blue dashed line presents the lower limiting factor as a result of the reflectivity coefficient of the mirrors, the solid black line with $0.05 \%$ notation highlights the typical limit of the relative standard uncertainties in measuring laser power at $10 \mathrm{~W}$ and above with conventional power meters [7].

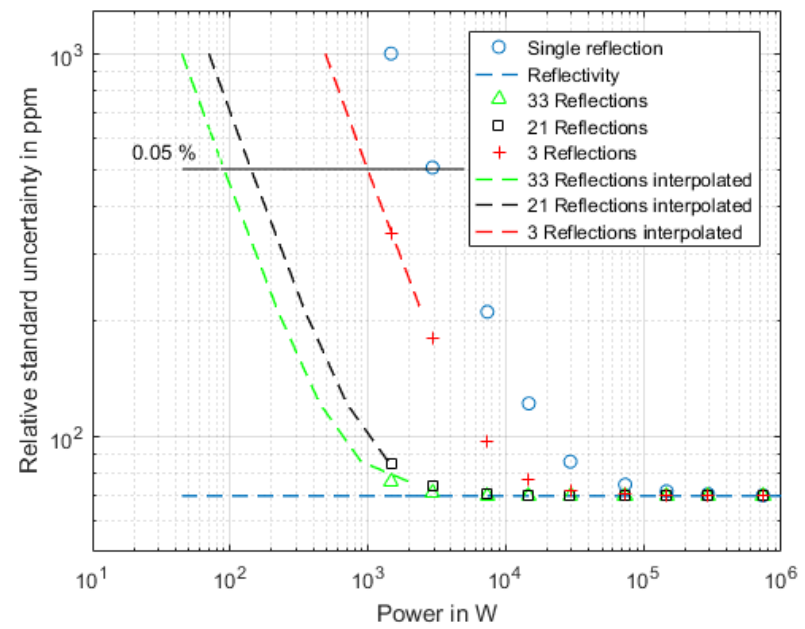

Figure 4: Estimated relative standard uncertainty of the optical power realisation by equations (5) and (6)

This method of establishing the traceability of the optical power measurements through the use of measurements of the photon momentum-generated forces and their further intercomparisons with the standards and procedures known from mass/force metrology is theoretically more direct and simplistic which may further improve and lower the measurement uncertainties at least in continuous wave optical power measurement.

\section{RESULTS}

Currently, we perform deliberately planned trial measurements in collaboration with Division 4 (optics) PTB, Braunschweig for establishing the traceability of the optical power measurements through the use of photon momentum-based force measurements and we compare the results with those performed by using a calibrated reference standard detector, Thermopile and Si-diode traceable to PTB's primary standard for optical power (the cryogenic radiometer) as shown in schematics in given in Figure 1.
The optical power values were used to calculate theoretically the expected photon momentumgenerated forces for comparison with the data from the measurements. In this configuration we can directly compare the reference for the force measurements and the reference for the optical power measurements, towards the SI-based traceable comparison (actually in future to be in reference to the Planck constant) of the force/mass and laser power references.

In Figure 5 we show one typical set of static forces and the corresponding laser power measurements.

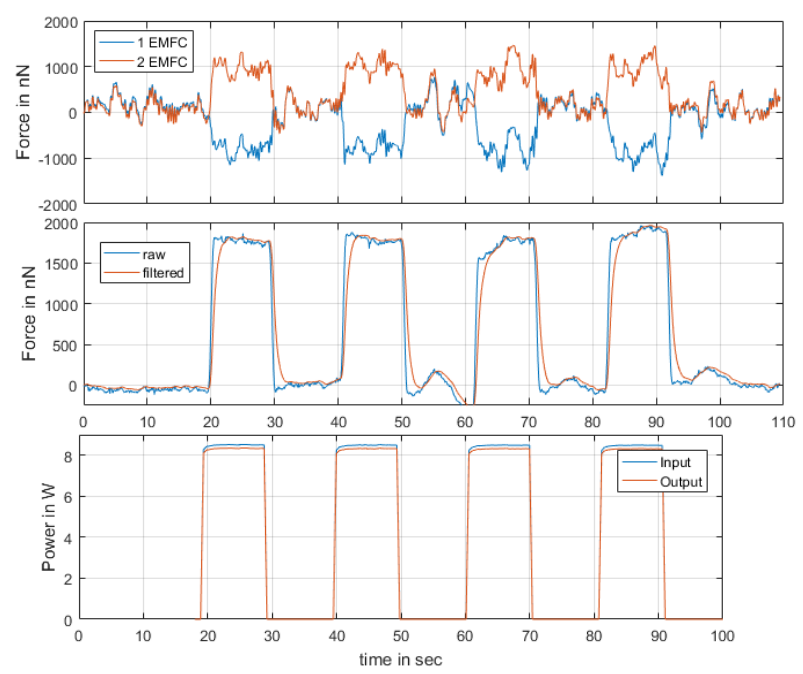

Figure 5: Example of the static force measurements with a periodically applied laser power (10 s) in the case of 33 reflections. Top - measured force signal from each balance. Middle - the difference signal (a feed backward averaging filter was chosen for the last 15 bins to filter raw data). Bottom - input and output power

From the raw data of the force measurements during the 33 reflections configuration (wavelength $532 \mathrm{~nm}$, mirror reflectivity 0.99995 , input power $8.5 \mathrm{~W}$, output power $8.3 \mathrm{~W}$ ), a mean value of $1873 \mathrm{nN}$ is obtained with a relative combined standard deviation of $1.71 \% \quad(32 \mathrm{nN})$. As a comparison, the standard uncertainty of the optical power measurements using the calibrated reference standard detector was $0.3 \%$. Furthermore, in this particular case, the mean value of the measured forces in comparison to the value calculated theoretically differs by $1.07 \%$, from all measurements this difference is within $3 \%$.

With these measurements at $10 \mathrm{~W}$ level using well established methods from mass/force metrology we demonstrate that upon the increase of the input power entering to the optical cavity and the number of reflections, the resulting uncertainties of generated small force values or vice versa the measurements of the input optical power can markedly be reduced. Thus, by collecting more experimental evidence at the edge of the small force measurements in static and dynamic regimes, improving the accuracy of the measurements, and 
eliminating further the systematic errors associated with the measurements, then the photon momentum-based force generation can serve more evidently as a superior method for obtaining accurate measurement of small (calibration) forces below $10 \mu \mathrm{N}$ through the traceable optical power measurements. Thereafter, by virtue of the same relation, the photon momentum-based force measurements can be used to develop viable, accurate and absolute optical power detectors / sensors / power meters at higher ranges with direct traceability to SI units. In accordance with the existing technical limits and instrumentation capabilities as well as the theoretical obtained relations, we can still improve the accuracy and the precision of the optical power and small force measurements by at least several orders of magnitude (ref. Figure 4).

\section{SUMMARY}

In this work we present photon momentumgenerated precision small force measurements in static and dynamic modes, in the range of forces below $10 \mu \mathrm{N}$ and above $100 \mathrm{nN}$, using a force sensor with about $20 \mathrm{nN}$ resolution. Static (considered to be below $0.2 \mathrm{~Hz}$ ) and dynamic (ranging from $0.2 \mathrm{~Hz}$ to $10 \mathrm{~Hz}$ ) modes of measurements are considered for different excitation signals with sine and square waveforms whose peak-to-peak amplitudes range from several hundred milliwatts up to several watts. We compare the measured forces in reference to the calculated values. The calculations of the theoretical data are made based on reference measurements of the optical power by calibrated reference standard detector, Thermopile and Si-diode traceable to PTB's primary standard for optical power (the cryogenic radiometer).

\section{ACKNOWLEDGEMENT}

This work is funded by DFG Projektnummer 409476492. The authors are grateful to colleagues from Institut für Prozessmess- und Sensortechnik and collaborators from PTB Division 4 for all fruitful discussions.

\section{REFERENCES}

[1] S. Vasilyan, T. Fröhlich, E. Manske, "Total momentum transfer produced by the photons of a multi-pass laser beam as an evident avenue for optical and mass metrology," Optics Express, vol. 25, pp. 20798-20816, 2017.

[2] E. Manske, T. Fröhlich, S. Vasilyan, "Photon momentum induced precision small forces: a static and dynamic check", Meas. Sci. Technol. vol. 30, 105004, 2019.

[3] G. A. Shaw, J. Stirling, J. Kramar et al., "Comparison of electrostatic and photon pressure force references at the nanonewton level", Metrologia, vol. 56, 025002, 2019.

[4] P. Williams et al., "Portable, high-accuracy, nonabsorbing laser power measurement at kilowatt levels by means of radiation pressure", Opt. Express, vol. 25, pp. 4382-92, 2017.

[5] P. A. Williams et al., "Meta-study of laser power calibrations ranging 20 orders of magnitude with traceability to the kilogram", Metrologia, vol. 57, 015001, 2020.

[6] C. Rothleitner, J. Schleichert, L. Günther et al., "The Planck-Balance - Using a fixed value of the Planck constant to calibrate $\mathrm{E}_{1} / \mathrm{E}_{2}$-weights," Meas. Sci. Technol., vol. 29, 074003, 2018.

[7] https://www.ptb.de/cms/ptb/fachabteilungen/abt4/ kalib.html, PDF Kalibrier-und Messmöglichkeiten der Abt. 4.

[8] OIML R 111-1: Weights of classes $E_{1}, E_{2}, F_{1}, F_{2}$, $\mathrm{M}_{1}, \mathrm{M}_{1-2}, \mathrm{M}_{2}, \mathrm{M}_{2-3}$ and $\mathrm{M}_{3}$ - Part 1: Metrological and technical requirements, International Organization of Legal Metrology, 2004.

[9] Super Mirror, $30 \mathrm{~mm}$ diameter, $532 \mathrm{~nm}$ wavelength, TFHSM-50C08-532, SIGMAKOKI Group. 